\title{
Radiosurgically treated acromegaly
}
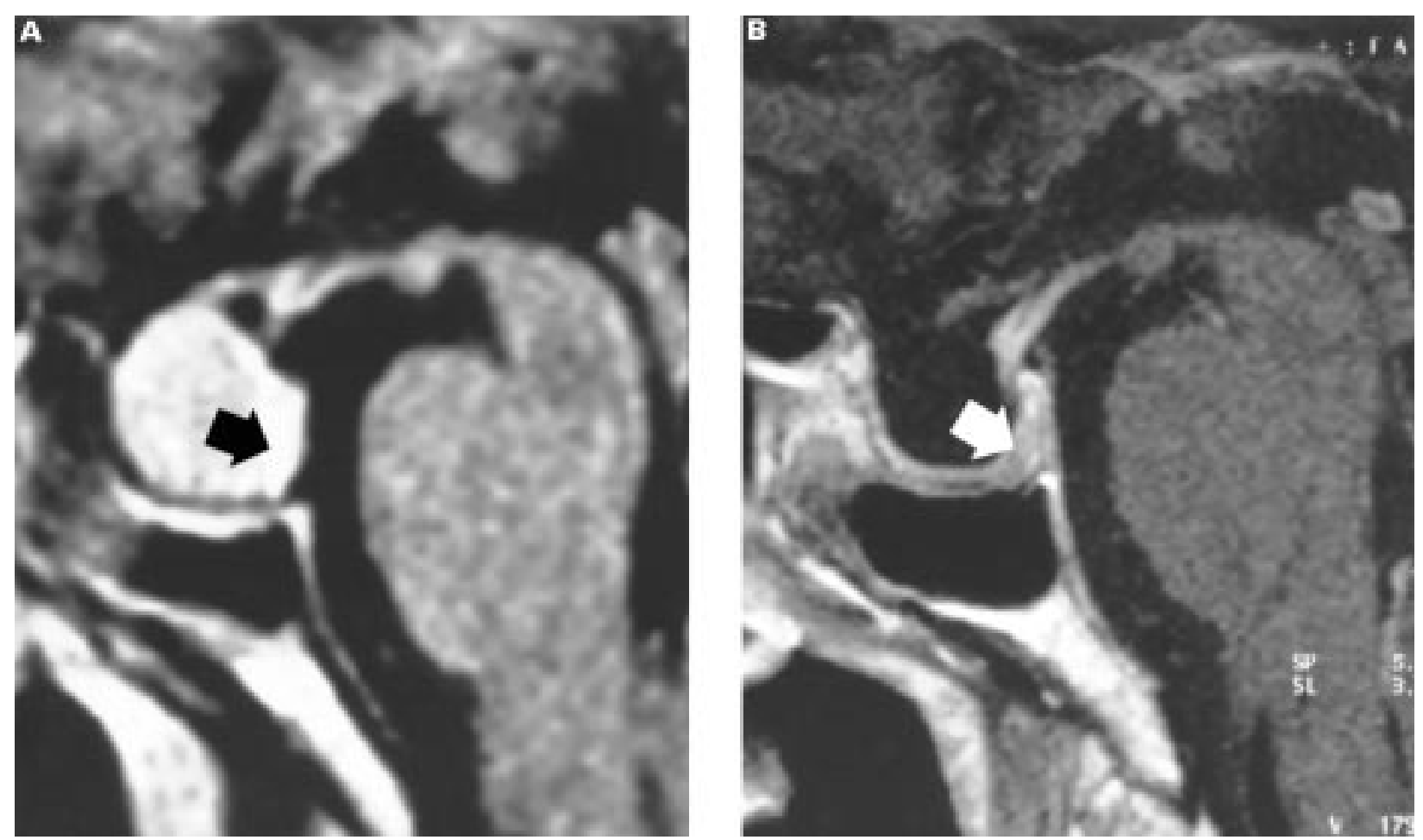

Gadolinium enhanced sagittal T1 weighted MRI. (A) Image before radiosurgery showing pituitary macroadenoma. The pituitary gland is displaced posteriorly (black arrow). (B) Image obtained 4 years after radiosurgery showing complete remission of the tumour. The normal pituitary gland is preserved (white arrow).

This 52 year old man presented with a 3 year history of acromegalic features. Endocrinological studies confirmed the diagnosis by showing raised serum concentrations of growth hormone $(\mathrm{GH} ; 76.8 \mathrm{ng} / \mathrm{ml})$ and insulin-like growth factor-1 (IGF-1; $1200 \mathrm{ng} / \mathrm{ml}$ ). Brain MRI disclosed a pituitary macroadenoma with suprasellar extension (figure A). As the patient had severe pulmonary emphysema which did not allow general anaesthesia, radiosurgery was chosen for the treatment modality. Using a gamma knife, more than 25 Gy of radiation was delivered in a single dose to the intrasellar part. The dose to the suprasellar part was tailored to keep the dose to the optic nerve and chiasm under 10 Gy. Four years after irradiation, the serum GH and IGF-I concentrations were normal $(2.6 \mathrm{ng} / \mathrm{ml}$ and $213 \mathrm{ng} / \mathrm{ml}$ respectively). Brain MRI showed disappearance of the tumour mass with preservation of the normal gland (figure B). He has been followed up for an additional 3 years, and has remained the same without optic neuropathy or hypopituitarism. Somatostatin analogue or bromocriptine was not administered throughout the course. Radiosurgery has been reported to be effective in the control of excess GH production in selected cases with pituitary adenoma and acromegaly, however, to our knowledge, this is a first case in which radiosurgery succeeded in complete resolution of the tumour.

HIROKI KURITA
SHUNSUKE KAWAMOTO
TAKAAKI KIRINO
Department of Neurosurgery,
Faculty of Medicine,
University of Tokyo,
Hongo, Bunkyo-ku,
Tokyo, Fapan

Correspondence to: Dr Hiroki Kurita, Neurochirurgische Universitätklink, Albert-Ludwings-Universität Freiburg, Breisacher Straße 64, D-79106, Freiburg, Germany Telephone 0049-761-2705006; fax 0049-761-2705008. 